\title{
Advertising Disclosures: Clear and Conspicuous or Understood and Used?
}

\author{
David W. Stewart and Ingrid M. Martin
}

\begin{abstract}
Although the frequency of disclosure usage has increased in recent years, adherence to individual disclosure regulations, such as those mandated under the "clear and conspicuous" standard guidelines, has declined or remained unchanged in the context of television advertising. Investigation of the cause of declining or static adherence levels would be useful to regulators and industry professionals to consider for advertising disclosures when designing and evaluating guidelines. This article offers a discussion of issues related to implementation of information disclosures on products and in marketing communications. The authors suggest that there are circumstances in which there is a need for alternatives to natural market mechanisms and message-focused regulation to ensure that consumers are well-informed. They also recommend that guidelines for disclosure focus on critical responses of consumers and media and message characteristics.
\end{abstract}

$\mathbf{N}$ atural competition and market mechanisms are often sufficient to ensure that consumers are well-informed. There are significant economic incentives for firms to inform consumers about the positive characteristics of their products and identify the shortcomings of competitors' products. The ability of marketers to inform consumers about the ways their products are superior to competitors' products is an important incentive for product innovation and improvement. However, there are occasions when market mechanisms do not ensure that consumers are informed about product characteristics that may be integral to their decision to purchase and use a given product. In such cases regulatory action may be required to ensure affirmative disclosure.

Although information disclosure is a simple idea and, at first brush, appears to be easy to implement, it is neither simple nor easy. In an effort to provide guidance to marketers and thereby facilitate more effective information disclosure, the Federal Trade Commission (FTC) developed guidelines for affirmative disclosure in 1970: the "clear and conspicuous" standard (CCS). These guidelines are intended to provision of direction to marketers about acceptable standards for disclosure, and these standards have been extended and amplified in various policy statements and orders since their inception (Hoy and Andrews 2004). Similar types of disclosure guidelines have been developed by other federal agencies, including the Food and Drug Administration (e.g., direct-to-consumer pharmaceutical advertising), the Environmental Protection Agency (e.g., mileage

David W. Stewart is Robert E. Brooker Professor of Marketing, Marshall School of Business, University of Southern California (e-mail: david.stewart@marshall.usc.edu). Ingrid M. Martin is Associate Professor of Marketing, Department of Marketing, College of Business Administration, California State University, Long Beach (e-mail: imartin@csulb.edu). The authors gratefully acknowledge the comments and suggestions of several JPP\&M reviewers, which contributed to the development and evolution of the article. and emission standards for cars), the Securities and Exchange Commission (e.g., corporate information), and various state government regulatory agencies.

It is gratifying that the use of disclosures in television advertising has increased over time (Hoy and Andrews 2004). However, it is also disconcerting that disclosure guidelines, such as the CCS, are so often ignored even as the frequency of disclosures in advertising has increased (Hoy and Andrews 2004; Hoy and Stankey 1993). The numbers of products and services that require disclosures and the complexity of what needs to be communicated have dramatically increased. This creates a significant burden on advertisers, consumers, and regulators and raises questions about how best to inform consumers and regulate marketing communications. In some cases, information disclosure alone may be insufficient to produce intended outcomes. However, affirmative disclosures, at least in some forms, may not always be necessary or even desirable, and they may produce outcomes that are contrary to the intended effects with respect to consumer response. Thus, it is useful to consider the potential roles and objectives of affirmative disclosure, including alternative strategies for disclosure, the roles of message timing and media type, measurement issues, and alternatives other than affirmative disclosure for achieving broader objectives related to consumer welfare.

This article draws on extensive prior literature to offer a discussion of the increasingly complex issues related to information disclosure. We examine the role of disclosure and the challenges that confront marketers and regulators in designing and assessing the effectiveness of consumer disclosure. Although we recognize the potential value of regulation of the form and content of information disclosures, we challenge assumptions that underlie such regulation and suggest alternatives to information disclosure that focus on the totality of the marketing communications plan and consumer response to marketing communication. We also identify potential unintended consequences of information disclosure and suggest that such consequences are especially likely to occur when requirements for disclosure fail to con- 
sider consumers' goals in obtaining information, ignore the complex interactions associated with multiple messages, and lack coordination among different regulatory agencies. We also consider issues that are related to the measurement and evaluation of consumers' response to information disclosure.

\section{Forms of Disclosure}

Affirmative information disclosures are a class of regulatory actions in which a marketer is required to inform consumers of certain facts about a product or service (Wilkie 1986). Affirmative disclosures take on a variety of forms that include provision of basic information about product characteristics, qualifications of product claims, risks of product usage, admonitions or recommendations about product purchase and/or use, and information about reducing or avoiding risk, as well as corrective advertising (Stewart and Martin 1994; Wilkie 1986).

A related form of regulation is prohibition of the use of certain claims (Wilkie 1985). Such withholding of information is an appropriate remedy when claims are false or cannot be adequately qualified. However, there are occasions when claims involve uncertain or probabilistic benefits and when information about such benefits may be useful to consumers. For example, Pappalardo and Ringold (2000) observe that evidence of the health benefits of polyunsaturated fats to the reduction of serum cholesterol and heart disease was available for decades before marketers were permitted to promote such benefits for their products. It is unclear that the withholding of such information from consumers, even when there remained some uncertainty, served the welfare of consumers. In hindsight, a properly qualified claim that provided consumers with information about the current state of scientific knowledge would have likely been beneficial to consumers. Thus, affirmative disclosure of qualifications or of uncertainty about product benefits represents an alternative to prohibition of the use of product claims that might otherwise be beneficial to marketers and informative for consumers.

\section{Objectives and Outcomes of Affirmative Information Disclosure}

In most circumstances, information disclosure is a means to an end rather than an end in itself. There are many potential objectives associated with information disclosure, including to protect marketers from future liability claims and to conform to specific laws and regulations, but a primary objective is a better informed consumer. It is often in the marketer's self-interest to disclose only information that is favorable with respect to its offerings, at least in the absence of penalties for withholding information. Although marketers also have incentives to reveal less favorable or negative information about competitors, the incentives are not always sufficiently large to overcome consequences of such disclosure. In general, consumers do not like negative advertising, marketers are loath to offer information that is negative relative to all competitors in a category, and one competitor's use of negative information in a market invites retaliation that may not be beneficial to any competitor. Pre- sentation of information that is negative, qualifies primary benefit claims, or warns of product risk also may distract consumers from primary selling messages, increase the costs of communication, and reduce the efficiency of the selling message. Thus, mandates for disclaimers, qualifiers, cautionary notes, and similar disclosures in advertising, product labels, collateral material, and elsewhere are sometimes necessary.

In a seminal set of papers, Wilkie (1982, 1983, 1985, 1986, 1987) examines more than 200 affirmative information disclosure orders by the FTC between 1970 and 1977. On the basis of his analysis of these cases, Wilkie (1985) identifies the following seven broad policy objectives: (1) an assertion of government presence and oversight in a market with an attendant increase in consumer confidence in market offerings, (2) an increase in awareness of specific information about a product or service, (3) an increase in beliefs about a product with respect to factual characteristic, (4) an increase in the degree of personalization of beliefs about a product, (5) a change in attitudes toward a product, (6) a change in behavioral intentions toward a product, and (7) a change in actual behavior with respect to a product. These objectives are varied and require quite different strategies for disclosure. The efficacy of affirmative disclosure relative to other means for influencing consumer behavior also varies considerably as these policy objectives move from creation of awareness to changes in consumer beliefs and behavior.

Even in the narrow domain of communication outcomes, there are many potential message objectives. The role of such message objectives also varies considerably depending on the broader policy outcomes sought. In his analysis, Wilkie (1985) also identifies nine specific message objectives of disclosures: (1) legibility, (2) prominence, (3) attention value, (4) changing consumer awareness, (5) changing consumer beliefs, (6) personalizing consumer beliefs, (7) changing consumer attitudes, (8) changing consumer intentions, and (9) changing consumer behavior. As Wilkie observes, the latter objectives, especially among the specific message outcomes, are difficult to achieve with disclosure alone. Outcomes related to changes in beliefs, attitudes, intentions, and behaviors are influenced by a wide array of factors in consumers' environments and individual differences.

Disclosure standards, such as those posed by the CCS, are useful, but they focus on the message characteristics and outcomes in Wilkie's (1985) lists of policy and message objectives. Such a focus has significant merit because, in general, the ensuring of attention to information is a prerequisite for changes in consumer beliefs, attitudes, and behavior. However, this focus on the message, especially in isolation from the broader world of the consumer, is also limiting. There is a need to consider the benefits and limitations of a focus on message characteristics and alternative forms of regulation that might serve as substitutes for or complements to such standards.

\section{Regulation of Message Characteristics}

The regulation of message characteristics has several advantages. First, there are well-known principles of communica- 
tion that can be applied to the design of disclosures. Second, it is relatively easy to determine compliance with standards related to the form and content of a message. There is no need to measure consumer response; any communication can be readily compared with the standard. This saves time and expense compared with the obtaining of measures of consumer response. Finally, to the extent that disclosures must appear in the same advertisement or other communications as the primary product claim, there is no need to consider the effects of multiple communications or media in assessing compliance. Noncompliance can be judged with respect to any single communication.

Mandated disclosure guidelines, such as the CCS, are useful in many cases, and disclosures have been highly successful in providing better or more complete information to consumers. For example, few consumers today are unaware that there are health risks associated with smoking or that mileage may vary when comparing Environmental Protection Agency mileage ratings on motor vehicles. In contrast, it is less clear that many consumers take the time and effort to comprehend and use the various detailed disclosures with which they are confronted, especially in contexts such as direct-to-consumer advertising for prescription drugs and financial products. At some point, the amount of information that must be communicated makes any individual message element less clear.

Direct-to-consumer pharmaceutical advertising provides an extreme but illustrative example of this problem. The opposite side of most direct-to-consumer print advertising usually includes detailed information in fine print. The amount of information offered is so large that it must be placed on a different page from the primary advertising message. Thus, even by the most generous standards, any specific information disclosed is neither clear nor conspicuous. Similarly, the limited duration of television commercials limits the ability of consumers to self-pace presentation of information for various products and services. This means that television provides limited opportunity for very detailed disclosure. This may be the reason that the CCS and other disclosure rules are ignored so frequently in television advertising (Hoy and Andrews 2004). Nevertheless, when it is not practical or even possible to adhere to mandated disclosure rules such as the CCS, in all or in part, there is a need for alternatives if consumers are to be appropriately informed.

\section{Determining Compliance or Measuring the Effects of Communication}

Wilkie (1985) observes that there are two contrasting views of the value of information. One view holds that information is valuable to consumers if it is present, regardless of whether consumers choose to use the information. The other view is that information has no value unless consumers use it. These views also offer competing definitions of effective disclosure. The view that information is of value when it is present is consistent with a definition of effective disclosure in terms of compliance with standards for specific message characteristics. A competing view suggests that the effectiveness of a disclosure should be assessed by how well consumers actually understand and use the relevant information provided in a disclosure.
However, there is a middle ground between these two views that turns on the meaning of "using" information. This middle ground has important implications for the definition and measurement of the effectiveness of disclosures. Information may be present and attended to by consumers, but consumers may also intentionally ignore or discount information that they regard as irrelevant, incomprehensible, or requiring too much effort. This circumstance may be the result of consumer characteristics, such as a lack of knowledge or an inability to read, but it is more likely to be the result of message characteristics, especially if the information is ignored or discounted by many target consumers.

Conversely, consumers may understand the presented information and choose to give it little or no weight when making decisions about purchasing and using products. Thus, the risk-averse consumer may give considerable weight to the perils of skydiving, whereas the risk-seeking consumer may give the same information little weight or consider risk to be a positive characteristic. Although both the risk-averse consumer and the risk-seeking consumer attend to and understand information about risk, they use this information differently. In both cases, the disclosure of risk information is effective in that the consumer understands and uses it to make an informed decision. An important implication of this middle view is that neither an assessment of compliance with a particular standard for disclosure nor knowledge of the ultimate behavior of the consumer provides much insight into the effectiveness of the disclosure.

It appears that regulatory agencies are beginning to embrace this middle ground. For example, a recent joint policy statement by the Federal Communications Commission and FTC regarding advertising for dial-around and other long-distance services states that "the disclosure should also be prominent enough so that typical consumers will actually read and understand it in the context of an actual ad" (Joint Federal Communications Commission/FTC Policy Statement 2000, p. 12, emphasis in original). This statement explicitly recognizes that it is the impact of a message on the consumer rather than the compliance with a specific form, per se, that is the objective of information disclosure.

\section{Including Consumer Response}

Wilkie (1982) argues that the success of regulatory actions should be measured in terms of consumer response. In discussing the use of corrective advertising, Wilkie, McNeill, and Mazis (1984, p. 26) observe, "Consumer effectiveness of corrective advertising has not been the primary concern of the orders issued to date." However, they also observe that there are reasons for the reluctance of marketers and regulators to address the success of disclosure in terms of consumer response, including the costs associated with obtaining measures of consumer response, the adversarial nature of relationships between marketers and regulators, the inherent complexity of consumer communications and their varied effects on consumers, and the costs of achieving some communications objectives. Another reason is that mere observation of the outcome of consumers' decisionmaking processes or uses of a product often is insufficient to determine whether consumers understood and complied 
with the information. Consumers' goals, existing product knowledge, prior beliefs, experience, and time constraints all influence attention to product information and its meaning (Stewart, Folkes, and Martin 2001).

\section{Consumers' Goals and Knowledge}

The understanding of consumers' motivation to attend to and use disclosure information is critical to any successful effort to inform. Consumers with minimal product familiarity are more likely to attend to information about what the product is and the benefits it provides than to warnings or disclosures about product use (Stewart and Martin 1994). Only after they identify what the product is and determine whether a product is goal relevant will consumers become interested in disclosures and warnings (Stewart, Folkes, and Martin 2001). At the other end of the familiarity continuum, consumers who are familiar with a product and any associated qualifications or warnings are likely to ignore a disclosure because they have already integrated the information. Thus, the degree of product familiarity influences consumers' goals in seeking product information and is an important factor in the consideration of how and when to present disclosure information effectively (see Alba and Hutchinson 1987).

A problem with focusing on the characteristics of communication messages is that such a focus ignores the extraordinary ways that consumers differ with respect to their goals and preferences and the ways such goals and preferences influence how they use information. As products and their associated information become more complex, there is greater opportunity for the individual variability of consumers to find expression in idiosyncratic responses to information. As the content of disclosures becomes more complex, it also becomes increasingly difficult to create useful and meaningful guidelines or standards for disclosure messages. It becomes more difficult to assume that compliance with a standard is related to how well consumers understand and use information, that is, such second-order outcomes of consumer awareness, knowledge, informed choice, and appropriate product use (Calfee 2001).

\section{Goals, Preferences, and Values}

Deeply embedded in any discussion of goals and preferences are questions of values. Not all consumers agree about the desirability of goals or share common preferences. Although there are strong data to support the proposition that wearing a seatbelt while driving reduces injuries, and most consumers know that wearing a seatbelt is a good idea, not all consumers comply. Consumers who are not predisposed toward wearing a seatbelt may be aware of the benefits of doing so and the risks associated with not doing so. Nevertheless, they may regard the avoidance of the discomfort and inconvenience of wearing a seatbelt as more important than the potential risks of not doing so. In the case of seatbelts, society has made a value judgment that the benefits of wearing a seatbelt outweigh the inconvenience, and individual preferences should be disregarded. Disclosure of the benefits of wearing a seatbelt may still be useful, but it lacks the power of regulation that focuses directly on the consumer behavior by making the use of seatbelts compulsory.
In many contexts, it is useful to consider whether there are alternatives that are more likely to produce specific outcomes than information disclosure alone. The FTC's Consumer Information Task Force (1979) identified many alternatives for influencing consumer behavior, ranging from those with a more cognitive character, such as disclosures, to alternatives that directly operate on consumer behavior, including active prohibition. Cognitive remedies preserve the right of consumers to make choices based on their own preferences, goals, and values, whereas behavioral remedies restrict consumer choice and impose the values and preferences of others on the individual consumer (Wilkie 1985). When society does not impose restrictions on consumer choice, information disclosure is especially important because it increases the likelihood that consumers' choices are based on actual preferences rather than on incomplete or incorrect information. However, the question remains: When and in what medium will disclosure be most useful to consumers?

\section{Message Timing}

Mandated disclosure guidelines assume that it is best to present disclaimers, qualifying information, or warnings in the same advertisement in which a product claim is made. There might be many circumstances in which the disclosure is short, simple, and easy for the consumer to understand and there is a single disclosure or relatively few disclosures. However, the longer and more complex the disclosure, and the greater the number of disclosures, the more important it is to consider the timing of disclosure and the attributes of the medium by which a disclosure can be delivered (Wilkie 1986, 1987). There are trade-offs that inevitably occur in the communication of and processing of complex, multidimensional information.

From a consumer welfare perspective, it is less important that any specific marketing communication includes all possible disclosures than that relevant disclosure occurs in a meaningful and salient way before the consumer acts. It is useful to consider whether consumers might be better served through strategic timing of disclosures to ensure that information is available at specific points in the purchase and product usage process when such information is most relevant to them.

Information may be more or less relevant and useful to a consumer depending on when and how it is received. Provision of information by a salesperson or customer-service representative at the time an order is placed may be more effective than highly detailed written disclosures for some consumer segments. Advertising disclosures that call attention to sources of additional information that the consumer can peruse at his or her leisure may be more useful than complex disclosures within an advertisement to some consumer segments. Some information may not be needed by or be relevant to the consumer at the time of product purchase but may be especially useful during product use. Usage instructions, product labels, product inserts, and other types of communications are likely to be relevant to the consumer during actual product use. Consumers also may be well served by external memory aids at the time of purchase or use even if disclosures are provided in other forms of communication (Wilkie 1986). 


\section{Media Effects}

Certain product claims, because of their complexity or the need for significant qualification, may not be appropriate for some media. The media influence the degree to which consumers attend to, comprehend, and use information (Stewart, Pavlou, and Ward 2002). The case of television provides a useful illustration of the limitations associated with particular media. Television advertising is an especially ineffective medium for providing consumers with information (Barwise and Ehrenberg 1988). It is a passive medium and among the less credible vehicles for advertising (Mital 1993). This type of advertising occurs in a short time frame and without prior notice, and thus the consumer cannot easily self-pace.

The very nature of television restricts what the advertiser can do in an effective and efficient manner. As Gerbner and colleagues (2002, p. 45) observe: "[C]ompared to other media, television provides a relatively restricted set of choices for a virtually unrestricted variety of interests and publics." An economic reality of advertising is that if mandated disclosures become the dominant element of the commercial, there will be less motivation for the advertiser to run the commercial. This is a negative outcome, because it reduces available information to consumers and eliminates a basis for competition among firms. Therefore, television advertising for a particular product might be restricted to creating awareness for a product and might include information that, for example, invites a call to a toll-free number, a call to an expert such as a doctor or a pharmacist, a visit to a retail outlet, or a visit to a Web site. (All the latter could contain detailed, customized information.) However, the placing of restrictions on the use of advertising claims that are important to the consumer and easily communicated because qualifying information cannot be easily and effectively provided may not be in the best interest of the consumer or the advertiser.

Marketers seldom depend on a single medium to communicate messages to consumers. It may also be the case that consumers will be better informed and better able to process information provided in disclosures when coordinated media are used (Wilkie 1987). Informing a consumer can include multiple media with different purposes. For example, a television commercial may create awareness of a potential benefit and direct the consumer to another source such as a customer-service representative, who would provide additional information to the consumer before purchase or consumption.

It is also useful to consider whether an advertisement is the best means for all types of information disclosure. Advertising is itself a weak communication tool that some consumers consider intrusive, unwanted, and low in credibility (Calfee and Ringold 1994; Shavitt, Lowry, and Haefner 1998). Thus, consumers may not perceive the vehicle within which a disclosure is embedded as an especially favorable, credible, or effective communication venue. Disclosures embedded in advertising are usually incidental to the primary purpose of the advertisement for both the advertiser and the consumer; the longer, more complex, and detailed the information in a given disclosure, the more likely it is that the disclosure will displace other content in the advertisement. Consumers are less likely to attend to any one specific piece of information in the disclosure, at least within any single exposure opportunity, given their tendencies to focus on goal-relevant information and discount goal-irrelevant information (Stewart and Martin 1994). In addition, the more the meaning of information in a disclosure depends on consumers' knowledge and goals, the less likely the disclosure alone will result in a well-informed consumer unless there are other means by which consumers understand the information and its relevance to their decisions. These latter tasks are matters of socialization and education.

\section{Coordinated Media and Substantiation of Effect}

A complement or an alternative to mandated disclosure guidelines, which are applied to each communication individually, is the use of coordinated media and message executions intended to ensure appropriate and relevant disclosure of information to the consumer before his or her purchase or use of the product (Wilkie 1987). Instead of focusing only on adherence to mechanical formulas that define attributes that are intended to make a disclosure clear and conspicuous, it may be more useful to require substantiation that disclosure has occurred or that a reasonable attempt at disclosure has been made before purchase or use. Such disclosures may not need to be in all advertising or take the same form in all media, a view that is potentially contrary to current FTC requirements, which hold that statements, label disclosures, or point-of-sale material do not necessarily correct a deceptive representation or omission. Nevertheless, media plans that chart the flow of information to consumers and demonstrate the points of contact where disclosures are provided might document that disclosure has occurred. Indeed, disclosure of information at demonstrably relevant points in consumers' purchase or usage process may be more effective than a requirement that specific information be included in every point of communication.

Evidence of disclosure also may be obtained by measuring consumer awareness of information through surveys. For example, simple tracking studies may be used to determine the percentage of consumers who are aware of the information contained in a particular disclosure. Although this places a greater burden on the advertiser, it might be preferable to filling a television commercial with so much information that it becomes neither a useful selling tool nor a useful vehicle for disclosing information, warnings, qualifiers, and/or disclaimers. Such survey measures are preferable, all other things being equal, because they focus on what the consumer has learned rather than the characteristics of the communication. Although the cost of such surveys may be prohibitive for smaller firms, for large, national advertisers the cost is far less than a single prime-time television commercial and, in many cases, less than the cost of a single four-color print advertisement in a popular national magazine. Such measures also can be obtained through copy testing that occurs before the actual use of advertising in the market, which provides evidence of proper disclosure before dissemination of advertising. This approach places a burden on the advertiser, but regulators should give considerable weight to such evidence, when it is present.

Evidence of a coordinated media campaign combined with survey research should be a sufficient substitute for 
guidelines such as the CCS. There are several advantages to this approach than to a focus on each individual message of the marketer. First, it enables the marketer to design an effective marketing program. This is consistent with Wilkie, McNeill, and Mazis's (1984) admonition that regulators get out of the business of creating advertising. Second, it is likely to result in a better informed consumer, because media and messages can be tailored to consumers' specific information needs that are the most salient at a given point in the purchase or product usage process. Third, this approach provides protection for advertisers from capricious litigation based on failure to adhere to mechanical standards for disclosure that may be neither appropriate nor relevant for a given product market. Fourth, the approach is consistent with best practices in advertising copy testing, which focus on understanding consumer response to advertising. Finally, by focusing on the consumer and effects of communication on consumer response, it is likely that unintended consequences of disclosure practices can be identified and remedied.

\section{Unintended Consequences of Information Disclosure}

Just as it is important to ensure that the intended objectives of information disclosure are obtained, it is also important to consider whether specific disclosure policies and practices carry unintended consequences. The unintended consequences of disclosures have only recently begun to receive attention (Stewart and Martin 1994). Indeed, Calfee (2001) observes that scarce attention has been paid to the effects of regulation of marketing practices. Unintended consequences are very real, however. If highly specific requirements for disclosure of information reduce the likelihood that advertisers will use television advertising, the consumer may not be well served. If so much information must be disclosed in a single advertisement that the consumer is distracted from the most relevant and important information, the consumer may not be well-informed. If consumers forgo the benefits of a product because disclosures cause them to underestimate its benefits relative to competitive alternatives or overestimate the risk of using the product, the consumer is not well served. For example, if the risks of immunization are incorrectly perceived to exceed the benefits of the vaccinepreventable disease, parents may forgo the substantial benefits associated with immunization (Flanagan-Klygis 2003).

Consumer misperception based on information disclosure becomes especially problematic in the context of complex information mandated by separate agencies for different purposes. Failure to coordinate information disclosures requirements can produce misleading results. A recent decision by the California Supreme Court (Dowhall v. SmithKline Beacham Healthcare 2004) confronted such a problem. In a decision related to the requirement that warnings under California's Proposition 65 be included on nicotine patches (in addition to the disclosures mandated by the Food and Drug Administration), the Court found that "over warning" consumers may have the effect of misleading them. The Court observed that a "truthful warning can be misleading or fail to communicate the facts necessary for the protection of users" (Summary, II 16) and that warnings of "remote" or "tentative" risks may dilute the force of "specific" and "necessary" warnings.

The Court's decision illustrates the growing recognition of the need for broader coordination of the information provided to consumers. Regulatory focus on a single piece of information for a single product ignores the broader information environment in which consumers purchase and use products. The burden of coordination is not unique to advertisers; it must also be shared by regulators.

Consumers are not well served if the presence of disclaimers, warnings, and other disclosures causes them to generally ignore or become indifferent to such information. Twerski and colleagues (1976) speculate that the frequency of warnings in the environment may contribute to a perception that certain types of warning should be ignored. Similarly, Breznitz (1984) offers empirical evidence that repeated exposure to a warning message without immediate consequences may lead to "false-alarm" effects, that is, a diminution in the effectiveness of warnings over time.

\section{Goals, Types of Information, and Unintended Effects}

There are many types of information that can be provided to a consumer. Much of the information provided in advertising focuses on product benefits and points of differentiation between the advertised product and competitive products. However, the types of information that disclosures provide consumers are typically more conceptual in character and serve to qualify claims or suggest something about the interaction between a product and its use (e.g., what happens when more or less of the prescribed amount of ibuprofen is taken). Products also come with instructions that provide procedural information that describes the specific steps to follow when using the product (e.g., "Take two tablets every 4 to 6 hours"). Research demonstrates that provision of product disclosures for both more risky and less risky consumer products may adversely affect compliance with instructions and cause consumers to discount the risks associated with misuse of certain consumer products, an unintended consequence of disclosures (e.g., Martin 2004; Martin and Folkes 2002). A possible solution is to provide disclosure information (especially for risky products) that is more vivid and to model the desired behavior in the advertising (Keller and Block 1997). This solution requires a flexible approach to disclosure that includes the use of coordinated media. Another potential solution is the creation of packaging that encourages or ensures compliance with disclosures and instructions. For example, instead of instructions merely informing consumers that they should wear gloves when using a product, gloves could be packaged with the product.

An understanding of how consumers' usage goals interact with disclosure information is critical. Some usage goals may promote noncompliance or misuse of a product. For example, a consumer may purposefully take a larger dose of an analgesic than recommended because he or she believes that this dose will result in the optimal outcome (e.g., faster, more complete relief) (Martin 2004). Product instructions that state, "Use as directed," when combined with a consumer's belief that the recommended dosage is the mini- 
mally effective dosage, may cause the consumer to conclude that "more is better." If regulations do not differentiate among information disclosures that are more or less useful and relevant to the consumer, the consumer may not be able to differentiate the characteristics of a product that are genuinely important from trivial information. This latter problem is especially acute in the context of warning and healthrelated disclosures (Block and Keller 1995). If everything is dangerous, and no distinctions are made about the degree of danger, consumers are not well served. Similarly, if claims are qualified, it is important that the qualification enables the consumer to make informed trade-offs.

If there are positive effects from disclosures for some consumers and negative effects for other consumers, that is a problem that cannot be easily dismissed. If the disclosure of a beverage's alcohol content makes the beverage more attractive to some consumers while informing other consumers of the content, there is a trade-off between the value of the information to society and making the product more attractive, perhaps to a segment likely to abuse or misuse the product. Full disclosure of content may be appropriate, but it is not without consequences.

Finally, disclosure may result in distortions in available market offerings. If some products are required to carry disclosures, but others are not, the very presence of the disclosures could drive the product with the required disclosure from the market. For example, if the amounts of lead in cosmetic products, such as eye shadow, are small enough that no warning is required when the product is sold separately, but warnings are required when several different products are bundled together, there is an incentive for producers and retailers to sell individual products. This has the effect of eliminating the benefits from bundling for the consumer.

Bettman, Payne, and Staelin (1986) comment on the importance of ensuring that consumers can make comparisons among competitive alternatives. If information disclosure is not consistent across all alternatives, such comparison will be difficult. Similarly, Baron (2004) suggests a strategy of providing comparative risk information in disclosures (risk communications) to help make risk more relevant to consumers as they make choices about which protective behaviors to undertake and which risks are acceptable. Another strategy he recommends is to provide information about risk that helps the consumer compare risk to the individual with the risk to target consumers and society at large; for example, " $15 \%$ of pregnant women that eat foods high in mercury have had negative effects on their pregnancy."

Unintended consequences of regulation are far more likely to be identified and addressed when the focus of information disclosure is on consumers' understanding and use of information rather than on the form and content of the message. As information about products becomes more complex and requires greater qualification, disclosures will, out of necessity, become more complex. Such complexity increases the likelihood of unintended consequences. This places a greater burden on both marketers and regulators to assess the impact of communication on the consumer rather than to rely solely on guidelines and standards for message presentation.

\section{Measures of Information Disclosure Effectiveness}

Assessment of the impact of communication on consumers requires the use of relevant measures of consumer response, the second-order effects of communication. There is no shortage of measures of the influence of marketing communications, and a significant amount of marketing research is devoted to the assessment of the effects and effectiveness of marketing communications (Stewart, Furse, and Kozak 1983; Stewart et al. 1985). The critical criteria for determining whether a disclosure is adequate include measures of the degree to which information is understood and used by consumers. Measures of understanding range from comprehension of specific claims to knowledge and beliefs about particular products and services. Measurement is not the issue because such measures are well-known and widely used.

There are differences in what people think they know and what they actually know. Subjective knowledge, or consumers' perception of how much they know, may be quite different from consumers' objective knowledge (Brucks 1985). The confidence of a consumer may be influenced far more by subjective knowledge, whereas objective knowledge may be more important in determining what information consumers seek and how they use it. The importance of these differences extends beyond the narrow issue of disclosure, and it is unrealistic and unfair to require advertisers to bear the burden of educating consumers and correcting false beliefs that the advertisers did not create. In general, this is recognized in the regulation of advertising. Nevertheless, it may be appropriate in certain circumstances to expect marketers to proactively engage in activities that promote the education of consumers with respect to the purchase and use of products. At the same time, it is important that regulators recognize the need for flexibility in the face of complex information disclosure requirements.

Although tools for the measurement of the influence of disclosures and other forms of communication are well established, the criteria for determining the success of communication are less obvious. If consumer response is to be an acceptable means for establishing that consumers have been informed, there is a need to define what consumers should understand and how many consumers should possess this understanding. For example, it may not be realistic to assume that consumers will understand, or even care to understand, detailed probabilistic information. Research has shown that consumers often misuse or misinterpret numeric probability (e.g., Windschitl 2002). In contrast, it may be sufficient for consumers to understand that a claim is uncertain or does not apply in all cases; it may also be sufficient that consumers understand that use of a product requires reasonable care and the exercise of precaution or involves risk. The critical issue is not whether the consumer can recite specific facts and detailed qualifications about a product but whether the consumer has sufficient understanding to make informed decisions that are not influenced by misleading or incomplete information (regardless of whether the basis of the misleading or incomplete information is the marketer or government regulation).

It is also unlikely that even the most determined marketer can obtain an understanding of a specific message among 
$100 \%$ of the target audience. However, it should be the case that most target consumers have a reasonable opportunity for exposure to important product information before purchasing or using a product. The acceptable standard for creating such opportunities will vary by product and should include recognition of the likely consequences that arise from uninformed consumers. An appropriate first benchmark may be the level of effort expended by the marketer to make other, more positive claims. This does not mean that, for example, because a marketer expends $\$ 20$ million to make a particular claim that an equivalent expenditure (or fraction thereof) occur in an effort to provide important qualifying information to consumers. It is well understood among marketers that media expenditures are an especially poor measure of the impact of marketing communications. However, demonstration of the use of a salient and meaningful message, when it is relevant to consumers, can be especially powerful. Measures of message salience and meaningfulness are common; media plans recognize the role of message timing and relevance.

Measures of the extent to which consumers use information are more difficult to identify and define. A consumer who reads, understands, and ignores a safety message has "used" the information. The weight a consumer attributes to a particular fact or piece of information is different from whether the consumer uses the information. However, the weight given to the information could be a good measure of appropriate information use for that target segment. Further research should investigate this possibility. Qualifying information should reduce the emphasis a consumer places on a product claim compared with the emphasis placed on an unqualified claim.

\section{Preferences, Regret, and the Effects of Information Disclosure}

A consumer's decision that yields unwanted results is not necessarily an indication that he or she was poorly informed. In some cases, a consumer may obtain a negative outcome because he or she received inaccurate or incomplete information, but this is not always the case. Consumers change decisions over time as well as with additional information. For example, a skier who breaks a leg on the black diamond run may regret having chosen to ski that run. However, if the skier knew the risks associated with the challenging run, he made an informed choice to ski the run. That the skier has broken his leg is not relevant to how well-informed the skier was before deciding to ski the run. Regret cannot be the basis for evaluating whether a disclosure is effective.

Much of the information available to consumers involves probability and uncertainty. The skier might not have skied the run if he knew that he would break a leg. Information disclosures can provide consumers with knowledge about the probability (or uncertainty) of positive and negative outcomes before the purchase and use of a product. Disclosures also may inform consumers about how to reduce or avoid negative outcomes and how to increase the likelihood of positive outcomes. Disclosures cannot inform consumers about all potential, uncertain, and specific outcomes in the future. Preferences change with new information, and consumers often experience regret, but this does not mean that a consumer was uninformed at the time of decision. The skier who says he would not have skied the run if he had known about the broken leg is using hindsight bias and information that was not available at the time the decision was made.

\section{Summary and Conclusion}

Simple is better, when it works. Standards for the form and content of information disclosures in advertising and in other marketing communications are important and useful in providing guidance to marketers and in increasing the likelihood that consumers will be fully informed about the products they purchase and use. Standards such as the CCS are especially useful for simple disclosures when there is reason to believe that the presence of disclosures will have the intended consequence of creating more informed consumers. Nevertheless, such standards are limited in their applicability to more complex disclosures and specific types of media. In addition, it is not always the case that compliance with standards produces the intended result of a more informed consumer, especially in the context of complex information and traditional media, such as television and print advertising. As advertisers aim to provide more information about complex topics, such as the health and nutritional benefits of components and ingredients of food products, it will become increasingly difficult to write a set of standards for the form and content of disclosures that must appear in every advertisement that makes a specific benefit claim. Thus, there is a need for an alternative to standards such as the CCS that still ensures that advertisers provide consumers with the information required for an informed choice.

A fundamental axiom of marketing is customer focus. Regulation of marketing communication should be no less consumer centric. The notion that the regulation of information disclosures includes explicit consideration of consumer response is not original; suggestions of such an approach have appeared in the marketing literature and elsewhere for more than three decades. This view has been adopted in practice, at least in some venues. Evidence based on consumer surveys or other measures of consumer response are common, if not the rule, in many actions brought under the Lanham Act. The increasing complexity of consumers' information environment with its attendant potential for unintended consequences makes the need for measures of consumer response to information disclosure especially important.

It is ironic that attempts to inform consumers more fully about the complexities of the products they purchase and use, the trade-offs they must make among competitive alternatives, and the caveats associated with product use make it more difficult to specify relevant and practical standards for the form and content of advertising disclosures. However, there are alternatives.

Demonstrations of consumer awareness of qualifications, limitations, warnings, and other information that is common in disclosures through empirical consumer research provide advertisers with a means for establishing that consumers are informed even in the absence of compliance with standards for the form and content of disclosures. Similarly, media plans that specifically recognize and provide for information disclosure that is especially relevant to consumer decision 
making and product use may be reasonable and perhaps more effective alternatives to guidelines that focus solely on message characteristics. Such consumer-centric approaches may be more effective and efficient than attempts to write and enforce standards that apply the full range of complex disclosures and media vehicles with which marketers deal. Thus, "understood and used" might be an especially appropriate complement or alternative to "clear and conspicuous."

\section{References}

Alba, Joseph W. and Wesley J. Hutchinson (1987), "Dimensions of Consumer Expertise," Journal of Consumer Research, 13 (4), 411-54.

Baron, Jonathan (2004), "Cognitive Biases, Cognitive Limits, and Risk Communication," Journal of Public Policy \& Marketing, 23 (Spring), 7-13.

Barwise, Patrick and Andrew Ehrenberg (1988), Television and Its Audience. Newbury Park, CA: Sage Publications.

Bettman James R., John W. Payne, and Richard Staelin (1986), "Cognitive Considerations in Designing Effective Labels for Presenting Risk Information," Journal of Public Policy \& Marketing, 5, 1-28.

Block, Lauren Goldberg and Punam Anand Keller (1995), "When to Accentuate the Negative: The Effects of Perceived Efficacy and Message Framing on Intentions to Perform a Health-Related Behavior," Journal of Marketing Research, 32 (May), 192-203.

Breznitz, S. (1984), Cry Wolf: The Psychology of False Alarms. Hillsdale, NJ: Lawrence Erlbaum Associates.

Brucks, Merrie (1985), "The Effect of Product Class Knowledge on Search Behavior," Journal of Consumer Research, 12 (June), $1-16$.

Calfee, John E. (2001), "Insights from Consumer Research on the Effects of Deceptive Advertising Regulations," in Handbook of Marketing and Society, Paul Bloom and Greg Gundlach, eds. Newbury Park, CA: Sage Publications, 421-35.

and Debra J. Ringold (1994), “The 75\% Majority: Enduring Consumer Beliefs about Advertising," Journal of Public Policy \& Marketing, 13 (Fall), 228-38.

Dowhal v. SmithKline Beacham Healthcare (2004), 04 C.D.O.S. 3259, April 15.

Flanagan-Klygis, Erin (2003), "Policy Forum 1: School Vaccination Laws," Virtual Mentor, Ethics Journal of the American Medical Association, 5 (November), 1-4.

FTC Consumer Information Task Force (1979), Consumer Information Remedies. Washington, DC: FTC.

Gerbner, George, Larry Gross, Michael Morgan, Nancy Signorielli, and James Shanahan (2002), "Growing Up with Television: Cultivation Processes," in Media Effects, 2d ed. Jennings Bryant and Dolf Zillman, eds. Mahwah, NJ: Lawrence Erlbaum Associates, 43-67.

Hoy, Mariea Grubbs and Andrews, J. Craig (2004), "Adherence of Prime-Time Televised Advertising Disclosures to the "Clear and Conspicuous' Standard: 1990 Versus 2002," Journal of Public Policy \& Marketing, 23 (Fall), 170-82.

_ and Michael J. Stankey (1993), "Structural Characteristics of Televised Advertising Disclosures: A Comparison with the FTC Clear and Conspicuous Standard," Journal of Advertising, 22 (2), 47-58.
Joint Federal Communications Commission/FTC Policy Statement (2000), For the Advertising of Dial-Around and Other LongDistance Services to Consumers, File No. 00-EB-TCD-1 (PS), March 1, [available at http://www.fcc.gov/os/2000/03/jpsada. pdf].

Keller, Punam Anand and Lauren Goldberg Block (1997), "Vividness Effects: A Resource-Matching Perspective," Journal of Consumer Research, 24 (December), 295-304.

Martin, Ingrid M. (2004), "The Impact of Risk Perceptions, Usage Goals, and Disclosures on Instructional Compliance," paper presented at AMA's Marketing and Public Policy Conference, Salt Lake City (May 20-22).

and Valerie S. Folkes (2002), "The Unintended Impact of Product Disclosures on Compliance with Instructions," paper presented at AMA's Marketing and Public Policy Conference, Atlanta (May 16-18).

Mital, Banwari (1993), "Public Assessment of TV Advertising: Faint Praise and Harsh Criticism," Journal of Advertising Research, 34 (1), 35-53.

Pappalardo, Janis and Debra Ringold (2000), "Regulating Commercial Speech in a Dynamic Environment: Forty Years of Margarine and Oil Advertising before the NLEA," Journal of Public Policy \& Marketing, 19 (Spring), 74-92.

Shavitt, Sharon, Pamela Lowry, and James Haefner (1998), "Public Attitudes Toward Advertising: More Favorable Than You Think," Journal of Advertising Research, 38 (July/August), $7-22$.

Stewart, David W., Valerie S. Folkes, and Ingrid M. Martin (2001), "Warnings, Disclosures, and Usage Instructions," in Handbook of Marketing and Society, Paul Bloom and Greg Gundlach, eds. Newbury Park, CA: Sage Publications, 355-71.

, David H. Furse, and Randall Kozak (1983), "A Descriptive Analysis of Commercial Copytesting Services," in Current Issues and Research in Advertising, Vol. 6, C. Martin and J. Leigh, eds. Ann Arbor: University of Michigan Business School, 1-44.

and Ingrid M. Martin (1994), "Intended and Unintended Consequences of Warning Labels," Journal of Public Policy \& Marketing, 13 (Spring), 1-19.

, Paul Pavlou, and Scott Ward (2002), "Media Influences on Marketing Communications" in Media Effects: Advances in Theory and Research, rev. ed., Jennings Bryant and Dolf Zillmann, eds. Hillsdale, NJ: Lawrence Erlbaum Associates, 353-96.

, Connie Pechmann, Srinivasan Ratneshwar, Jon Stroud, and Beverly Bryant (1985), "Methodological and Theoretical Foundations of Advertising Copy Testing: A Review," in Current Issues and Research in Advertising, J.H. Leigh and C.R. Martin, eds. Ann Arbor: University of Michigan Business School, 1-74.

Twerski, A.D., A.S. Weinstein, W.A. Donaher, and H.R. Piehler (1976), "The Use and Abuse of Warnings in Products Liability: Design Defect Litigation Comes of Age," Cornell Law Review, 61 (4), 495-540.

Wilkie, William L. (1982), "Affirmative Disclosure: Perspectives on FTC Orders," Journal of Marketing \& Public Policy, 1, 95-110.

(1983), "Affirmative Disclosure at the FTC: Theoretical Framework and Typology of Case Selection," Journal of Marketing \& Public Policy, 2, 3-15. 
(1985), "Affirmative Disclosure at the FTC: Objectives for the Remedy and Outcomes of Past Orders," Journal of Public Policy \& Marketing, 4, 91-111.

(1986), "Affirmative Disclosure at the FTC: Strategic Dimensions," Journal of Public Policy \& Marketing, 5, 123-30.

(1987), "Affirmative Disclosure at the FTC: Communication Decisions," Journal of Public Policy \& Marketing, 6, $33-42$.
, Dennis L. McNeill, and Michael B. Mazis (1984), "Marketing's 'Scarlet Letter': The Theory and Practice of Corrective Advertising," Journal of Marketing, 48 (Spring), 11-31.

Windschitl, Paul D. (2002), "Judging the Accuracy of a Likelihood Judgment: The Case of Smoking Risk," Journal of Behavioral Decision Making, 15 (1), 19-35. 